\title{
The family of apoptosis-stimulating proteins of p53 is dysregulated in colorectal cancer patients
}

\author{
LIBIN YIN ${ }^{1}$, YUYANG LIN ${ }^{1}, \mathrm{XU}_{\text {WANG }}{ }^{1}$, YANZHUO $\mathrm{SU}^{2}, \mathrm{HAN} \mathrm{HU}^{1}$, \\ $\mathrm{CHAO} \mathrm{LI}^{1}$, LEI WANG $^{1}$ and YANFANG JIANG ${ }^{3,4}$ \\ ${ }^{1}$ Department of Colorectal and Anal Surgery, The First Hospital of Jilin University, Changchun, Jilin 130021; \\ ${ }^{2}$ Department of Gastrointestinal Colorectal and Anal Surgery, China Japan Union Hospital of Jilin University, Changchun, \\ Jilin 130000; ${ }^{3}$ Genetic Diagnosis Center, The First Hospital of Jilin University; ${ }^{4}$ Key Laboratory of Zoonosis Research, \\ Ministry of Education, The First Hospital of Jilin University, Changchun, Jilin 130021, P.R. China
}

Received March 13, 2017; Accepted January 16, 2018

DOI: $10.3892 / \mathrm{ol} .2018 .8151$

\begin{abstract}
The apoptosis-stimulating protein of p53 (ASPP) family is a newly identified family protein including ASPP1, ASPP2 and inhibitor of ASPP (iASPP), by which the tumor protein 53 (TP53)-mediated apoptotic process is selectively regulated. Downregulation of ASPP1/ASPP2 and upregulation of iASPP were revealed to be associated with a poor prognosis and metastasis in several types of cancer. However, to the best of our knowledge, the expression of ASPP in colorectal cancer (CRC) has not previously been investigated. The present study analyzed ASPP expression in human CRC tissues with multiple clinical and pathological profiles. A total of 41 patients diagnosed with CRC were enrolled in the present study. The expression of ASPP was detected by immunohistochemistry, immunofluorescence and reverse transcription-quantitative polymerase chain reaction. In addition, the variation in ASPP expression was examined in a number of pathological groups. The associations among ASPP expression, and the expression of TP53, plasma carcinoembryonic antigen (CEA) levels and $\alpha$-fetoprotein (AFP) levels were also investigated. ASPP1 and ASPP2 expression was significantly reduced, while iASPP expression was elevated in CRC samples compared with expression in adjacent non-cancerous tissues. Downregulation of ASPP1 was detected in the TP53-positive group compared with
\end{abstract}

Correspondence to: Dr Yanfang Jiang, Genetic Diagnosis Center, The First Hospital of Jilin University, 71 Xinmin Street, Changchun, Jilin 130021, P.R. China

E-mail: yanfangjiang@hotmail.com

Dr Lei Wang, Department of Colorectal and Anal Surgery, The First Hospital of Jilin University, 71 Xinmin Street, Changchun, Jilin 130021, P.R. China

E-mail: leiwang1967@163.com

Key words: colorectal cancer, apoptosis-stimulating protein of p53 1, apoptosis-stimulating protein of p53 2, inhibitor of apoptosis-stimulating protein of $\mathrm{p} 53$, tumor protein $\mathrm{p} 53$, carcinoembryonic antigen, $\alpha$-fetoprotein the TP53-negative group. The increase in iASPP expression was correlated with the grade of malignancy, but not with regional lymph node status or metastases. The expression of ASPP2 was negatively correlated with plasma CEA levels. The results of the present study, not only enrich CRC epidemic and pathological data, but also provide valuable indices for CRC clinical treatment and prognosis.

\section{Introduction}

Colorectal cancer (CRC) is the third most common cancer with $\sim 1.4$ million cases (10\% of all cancers) diagnosed every year globally (1). CRC is also the second leading cause of cancer-associated mortality with $\sim 700,000$ mortalities every year (1). At present, the pathogenesis of CRC remains unclear; however, CRC is primarily associated with old age and certain lifestyle factors, including drinking and preserved food, whereas a small proportion of CRC is associated with underlying genetic disorders. Certain inherited genetic disorders, including inflammatory bowel disease (Crohn's disease and ulcerative colitis) and familial adenomatous polyposis, are considered risk factors for CRC (1). Furthermore, there are other factors that also increase the risk of CRC, including diet (consumption of preserved food), obesity, smoking and a lack of physical activity (2).

As with other types of cancer, the mechanism of pathogenicity in CRC is fundamentally a disorder of uncontrolled cellular proliferation. In general, the inactivation of tumor suppressors, combined with the alteration of DNA repair genes, results in proto-oncogene mutations to produce oncogenes, which give rise to abnormal cellular proliferation and invasion (3). Tumor protein p53 (TP53) serves a crucial role in multicellular organisms in the prevention of tumor formation through regulating apoptosis, cell cycle and DNA repair processes (4-6). Different cellular stresses, including hypoxia, ionizing radiation, DNA damage and chemotherapeutic drugs, are able to stimulate the activation of TP53. Conversely, TP53 activity may be downregulated through TP53 gene mutations (loss of function mutations), alterations of upstream factors, or modifications of the downstream components that mediate TP53 signals (7). It has been reported that the TP53 mutational frequency is $5-21 \%$ in patients with breast cancer depending 
upon household income (8). High income patients may acquire fewer p53 mutations compared with low income patients (8). Approximately 50\% of TP53 mutation result in TP53 losing its (antitumor) activity (9). Previous studies have demonstrated that the degree of TP53 gene mutation is directly associated with the Dukes' stage, a staging method prognostically relevant to CRC, which includes the differentiation grade, extent of local invasion, liver and lymph node metastasis, and the prognosis of patients with CRC $(9,10)$.

Apoptosis-stimulating protein of p53 (ASPP) has recently been identified as a family of proteins with three members, ASPP1, ASPP2 and iASPP, which selectively regulate the TP53-mediated apoptotic process. These proteins commonly share an ankyrin repeat domain, a SRC Homology 3 domain and a poly-proline-rich domain at the C-terminus. ASPP1 and ASPP2 are pro-apoptotic factors, whereas iASPP has an anti-apoptotic effect (4). ASPP2 was revealed to be downregulated in several types of cancer, including choriocarcinoma (11), human acute leukemia (12), pancreatic cancer cells (13), pituitary adenoma, gastric cancer $(14)$, lung cancer $(15,16)$ and diffuse large B-cell follicular center lymphoma (17). It was reported that patients with cancer in which ASPP2 is downregulated exhibit metastasis and a poor prognosis $(11-13,17)$. Furthermore, iASPP expression is increased in non-small cell lung cancer, hepatocellular carcinoma and cervical adenocarcinoma, and is associated with a poor prognosis in these types of cancer $(15,18,19)$.

Although several reports have studied the role of ASPP in cancer prognosis (11-13,15,17-19), studies investigating the association between ASPP and CRC prognosis are limited. The present study investigated the expression profiles of ASPP1, ASPP2 and iASPP in 41 samples collected from CRC patients with different pathological conditions. The results of the present study will provide valuable pathological evidence to evaluate the prognosis of $\mathrm{CRC}$ in the clinic and to improve the treatment options for CRC.

\section{Materials and methods}

Clinical samples. Samples were collected from 41 patients pathologically diagnosed with CRC, including 20 males and 21 females, with a median age of 64 years (range, $41-86$ years) at the First Hospital of Jilin University (Changchun, China). CRC tissue samples and adjacent non-cancerous tissue samples ( $>5 \mathrm{~cm}$ from the edge of tumor) were obtained by surgical resection between June 2014 and April 2015. One part of the resected tumor and adjacent non-cancerous tissue was quickly frozen in liquid nitrogen and stored in a $-80^{\circ} \mathrm{C}$ freezer, while the other part was fixed with formalin for immunohistochemical analysis. The study research proposal was approved by the Medical Ethics Committee of the First Hospital of Jilin University, and written informed consent was obtained from each patient. The pathological classifications of these 41 samples are summarized in Table I.

According to the experimental results, the experimental data were divided into two groups: A TP53-positive group and a TP53-negtive group. Patients were additionally classified into early (Stage I+II) and advanced (Stage III+IV) groups, as well as $\mathrm{N} 0 / \mathrm{Nx}(\mathrm{x} \neq 0)$ groups, M0/M1 groups, good/moderate/poor histological grade groups and
Table I. Pathological classification of human colorectal cancer.
Clinical and pathological profile

Early/advanced (TNM I+II/III+IV) ${ }^{\mathrm{a}}$

Tumor topography $(\mathrm{T} 1 / \mathrm{T} 2 / \mathrm{T} 3 / \mathrm{T} 4)^{\mathrm{b}}$

Regional lymph node metastasis

(N0/Nx, $\mathrm{x} \neq 0)^{\mathrm{c}}$

Distant metastasis (M0/M1 $)^{\mathrm{d}}$

TP53 expression (Positive/negative) ${ }^{\mathrm{e}}$
Patient number

$11+7 / 14+9$

$5 / 9 / 16 / 11$

$19 / 22$

$10 / 31$

$15 / 26$
The colorectal cancers samples were classified based upon the TNM staging system (32). ${ }^{a}$ Classification based on the clinical course; ${ }^{\mathrm{b}}$ classification based on the size and/or extent of the primary tumor; ${ }^{c}$ classification based on whether $(\mathrm{Nx})$ or not (N0) the cancer has migrated to the regional lymph nodes; ${ }^{\mathrm{d}}$ classification based on whether (M1) or not (M0) the CRC exhibited distant metastases. ${ }^{\mathrm{C}} \mathrm{Classification}$ based on the TP53 expression. TNM, Tumor-Node-Metastasis; TP53, tumor protein $\mathrm{p} 53$.

colon/rectal groups based upon the Tumor-Node-Metastasis staging criteria of the American Joint Cancer Committee (20).

Immunohistochemistry (IHC). The TP53 and ASPP expression levels of the 41 patient tissue samples were detected by immunohistochemical staining. Briefly, the CRC and adjacent non-cancerous tissue samples were fixed with $10 \%$ formalin for $24 \mathrm{~h}$ at room temperature, embedded in paraffin and sliced into $4 \mathrm{~mm}$ thick sections, which were used for IHC and immunofluorescence (IF) staining analyses. The paraffin-embedded sections were deparaffinized by heating for $1 \mathrm{~h}$ at $60^{\circ} \mathrm{C}$, then washing with xylene for 15 min twice, and rehydrated in a descending alcohol series $(100,100,95,85$ and $75 \%)$. Antigen retrieval was performed by boiling, followed by incubation with citrate buffer $(0.01 \mathrm{M}$, $\mathrm{pH}$ 6.0) at room temperature for 2 min (repeated 5 times). Then, it was cooled to room temperature and the sections were washed with PBS for 5 mins 3 times. Subsequently, the endogenous peroxidase activity was inactivated with $3 \%$ hydrogen peroxide for $40 \mathrm{~min}$ at room temperature. Following blocking with 5\% donkey serum obtained from healthy animals for $40 \mathrm{~min}$ at room temperature, the sections were incubated with a 1:200 diluted mouse TP53 monoclonal antibody (cat. no. ZM-0405; Beijing Zhongshan Jinqiao Biotechnology Co., Ltd., Beijing, China), a 1:800 diluted mouse ASPP1 monoclonal antibody (cat. no. A4355; Sigma-Aldrich; Merck KGaA, Darmstadt, Germany), a 1:800 diluted mouse ASPP2 monoclonal antibody (cat. no. A4480; Sigma-Aldrich; Merck KGaA) or a 1:800 diluted rabbit iASPP polyclonal antibody (cat. no. ab34898; Abcam, Cambridge, MA, USA) overnight at $4^{\circ} \mathrm{C}$. The next day, sections were washed twice with phosphate-buffered saline (PBS), and then the pre-stained slice was incubated with the appropriate biotinylated secondary antibody for $1 \mathrm{~h}$ at room temperature. Biotin-labeled goat anti-mouse secondary antibody (cat. no. SP-0024; Beijing Biosynthesis Biotechnology Co., Ltd., Beijing, China; ready-to-use dilution) was used for TP53, ASPP1 and ASPP2 and biotin-labeled goat anti-rabbit secondary antibody (cat. no. SP-0023; Beijing Biosynthesis Biotechnology Co., Ltd.; ready-to-use dilution) was used for iASPP. The sections were washed three times with PBS. Streptavidin-peroxidase was 
reacted for $5 \mathrm{~min}$ at room temperature. Subsequently, the target protein was developed by freshly prepared 3,3'-diaminobenzidine reagent (Beijing Zhongshan Jinqiao Biotechnology Co., Ltd.). Finally, the sections were counterstained with hematoxylin for $2 \mathrm{~min}$ at room temperature, dehydrated through an ethanol gradient $(50,70,80,90,95,100$ and $100 \%)$ and sealed with neutral gum. The target protein was subsequently observed at a x200 magnification under a BX51 optical microscope (Olympus Corporation, Tokyo, Japan).

Immunofluorescence (IF). The paraffin-embedded sections were deparaffinized by heating for $1 \mathrm{~h}$ at $60^{\circ} \mathrm{C}$, then washing with xylene for 15 min twice, rehydrated in a descending alcohol series $(100,100,95,85$ and $75 \%)$ and antigen retrieval was performed by boiling, followed by incubation with citrate buffer (0.01 M, pH 6.0) and at room temperature for $2 \mathrm{~min}$ (repeated 5 times). Following retrieval, the slides were maintained at room temperature and were treated with $0.1 \%$ protease $\mathrm{K}$ to expose the antigen. The sections were subsequently blocked using $5 \%$ bovine serum albumin for $60 \mathrm{~min}$ at room temperature and were incubated with the appropriate primary antibodies (same as those used for IHC) overnight at $4^{\circ} \mathrm{C}$. Following rewarming at $37^{\circ} \mathrm{C}$ for $1 \mathrm{~h}$, the sections were washed in PBS for 5 min three times and were further incubated with fluorescence-conjugated secondary antibodies (donkey anti-mouse-A488, cat. no. ab150105 and donkey anti-rabbit-Tritc, cat. no. ab6799; both Abcam) at a 1:800 dilution for $30 \mathrm{~min}$ at room temperature. Following washing with PBS three times, the sections were stained with $0.001 \%$ 4',6-Diamidino-2-phenylindole dihydrochloride (Sigma-Aldrich; Merck KGaA) for $10 \mathrm{~min}$ at room temperature to stain the nuclei. The fluorescence-stained target protein was visualized using an Olympus FV1000 fluorescent microscope (Olympus corporation; magnification, $\mathrm{x} 200$ and $\mathrm{x} 400$ ). The omission of the primary antibodies was used as a negative control in all IF experiments. Quantification of the ASPP1, ASPP2 and iASPP expression was performed by measuring the total fluorescence intensity of the positively stained area.

$R N A$ preparation and reverse transcription-quantitative polymerase chain reaction $(R T-q P C R)$. Total RNA was extracted from the frozen tissue specimens using Total RNA Extractor (Sangon Biotech Co., Ltd., Shanghai, China). The RNA was reverse transcribed into cDNA using an AMV First Strand cDNA Synthesis kit (Sangon Biotech Co., Ltd.). The mixture of total RNA, random Primer $\mathrm{p}(\mathrm{dN})_{6}$ and rnase-free $\mathrm{ddH}_{2} \mathrm{O}$ was bathed at $70^{\circ} \mathrm{C}$ for $5 \mathrm{~min}$, and then placed in an $0^{\circ} \mathrm{C}$ ice bath for $10 \mathrm{sec}$. Then $5 \mathrm{X}$ reaction buffer, dNTP $\operatorname{mix}(10 \mathrm{mmol} / \mathrm{l})$, Rnase inhibitor (20 U/l) and AMV reverse transcriptase (10 U/l) were added into the mixture at $37^{\circ} \mathrm{C}$ for $5 \mathrm{~min}, 42^{\circ} \mathrm{C}$ for $60 \mathrm{~min}$ and $70^{\circ} \mathrm{C}$ for $10 \mathrm{~min}$ in order to synthesize the cDNA. RT-qPCR analysis was performed in a Light Cycler 480 (Roche Diagnostics, Basel, Switzerland) using SG Fast qPCR Master mix (BBI Solutions, Cardiff, UK). The GAPDH cDNA was employed as an internal control for each sample. ASPP expression was normalized using the $2^{-\Delta \Delta C q}$ method (21). The 40 cycles thermocycling conditions were: $95^{\circ} \mathrm{C}$ for $7 \mathrm{sec}, 55^{\circ} \mathrm{C}$ for $10 \mathrm{sec}$ and $72^{\circ} \mathrm{C}$ for $15 \mathrm{sec}$. All the primers used in RT-qPCR are as follows: GAPDH forward, 5'-TGGGTGTGAACCATGAGAAGT-3' and reverse, 5'-TGA GTCCTTCCACGATACCAA-3'; ASPP2 forward, 5'-GTGCTG CCTCATGTAACAACG-3' and reverse, 5'-GTAGCCTTCCTC
CATTTCCTC-3'; ASPP1 forward, 5'-CAGTGTATGGTAAGC CCGTTTT-3' and reverse, 5'-TGGACAGTGACCCGTGAA GA-3'; and iASPP forward, 5'-TGCCTACCACCATCATCA CAT-3' and reverse, 5'-GACCAATGTTTCCCACCCA-3.

Carcinoembryonic antigen (CEA) and $\alpha$-fetoprotein (AFP) assay. The concentrations of plasma CEA and AFP in patient samples were determined using an ADVIA Centaur XP immunoassay system (Siemens AG, Munich, Germany).

Statistical analysis. Two independent variables were analyzed using the Mann-Whitney U test, comparisons among multiple groups were performed using the Kruskal-Wallis test, and Pearson's correlation analysis was used to compare the associations. Statistical analyses were performed using the SPSS Version 16.0 statistical software package (SPSS, Inc., Chicago, IL, USA). $\mathrm{P}<0.05$ was considered to indicate a statistically significant difference.

\section{Results}

Altered ASPP expression in human CRC tissues. The protein expression of ASPP1, ASPP2 and iASPP was detected in human CRC specimens using IHC (Fig. 1A). The corresponding adjacent non-cancerous tissues were used as controls (Fig. 1A). The expression of ASPP proteins was detectable in normal epithelium, lamina propria and glands tissues in the CRC tissues, as well as the corresponding adjacent non-cancerous tissues. The results of the present study demonstrated that ASPPs are distributed in the nucleus and cytoplasm; however, they were more abundant in the cytoplasm. Quantification analysis of ASPPs in the IHC samples revealed that ASPP1 and ASPP2 proteins were expressed at significantly low levels $(\mathrm{P}<0.05)$, while iASPP protein expression was significantly upregulated $(\mathrm{P}<0.05)$ in the CRC samples compared with the adjacent non-cancerous tissues (Fig. 1A). In order to confirm these observations, the human CRC samples and the adjacent non-cancerous tissues were further analyzed using IF staining (Fig. 1B). IF staining results were consistent with the IHC data, whereby the expression of ASPP1 and ASPP2 was considerably downregulated and the expression of iASPP was significantly upregulated in CRC samples, compared with the adjacent non-cancerous controls $(\mathrm{P}<0.05)$. In order to further evaluate whether alteration in the protein levels of ASPPs in CRC is associated with RNA deregulation, the mRNA levels of ASPPs in human CRC samples were examined using RT-qPCR. As demonstrated in Fig. 1C, mRNA levels of ASPP1 and ASPP2 were decreased in CRC samples compared with expression adjacent non-cancerous tissues, whereas iASPP RNA expression was elevated in $\mathrm{CRC}$ tissues $(\mathrm{P}<0.05)$.

ASPP1 expression was significantly decreased in the TP53-positive CRC group. ASPPs are a protein family that regulates the apoptotic process; ASPP1 and ASPP2 activate apoptosis, while iASPP inactivates apoptosis. In order to investigate the association between ASPP and TP53 in human CRC tissues, the human CRC specimens were divided into TP53-positive ( $n=15$; Fig. 2A) and TP53-negtive (n=26; Fig. 2B) groups by IHC staining. The TP53 level of the corresponding adjacent non-cancerous tissues in IHC was used as a negative control (Fig. 2C). The ASPP levels were subsequently determined 

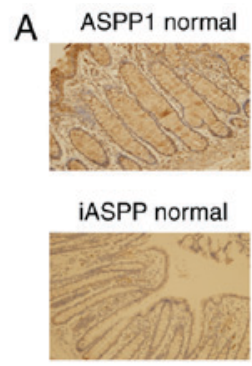

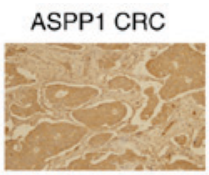

iASPP CRC

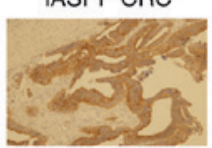

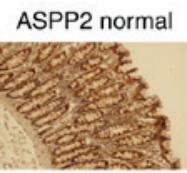
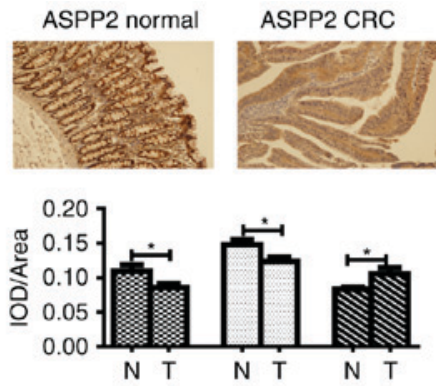

$\mathrm{ED}$ ASPP1 ASPP2 $\triangle$ iASPP
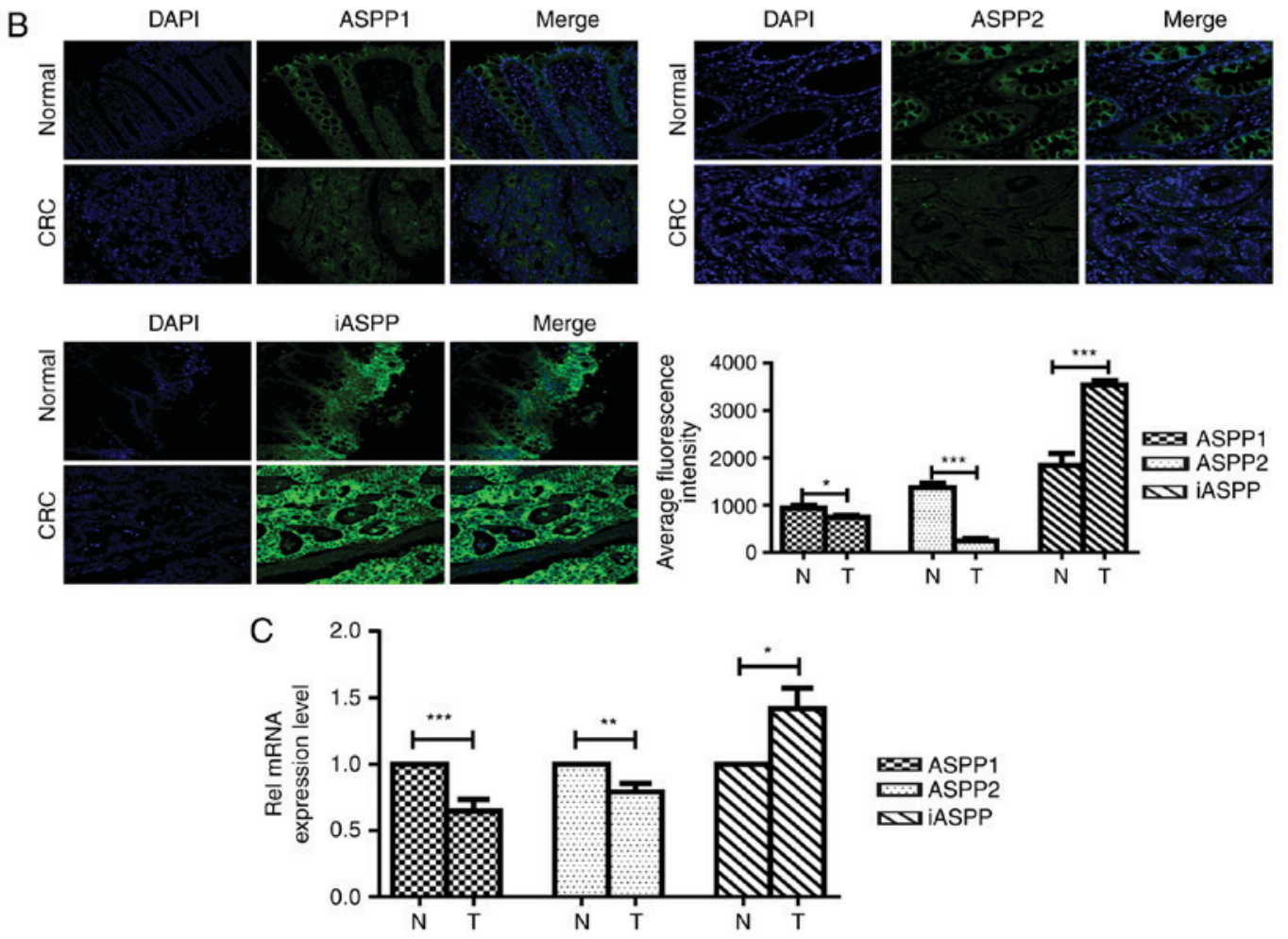

Figure 1. Alteration in ASPP expression in human CRC and corresponding adjacent non-cancerous tissues. (A) ASPP expression in CRC and corresponding adjacent non-cancerous tissues was detected by immunohistochemistry using ASPP1, ASPP2 and iASPP antibodies. The average IOD of ASPP1 (cross), ASPP2 (open) and iASPP (slash) in IHC stains of CRC samples (T) and adjacent non-cancerous controls (N) were statistically analyzed. (B) ASPP1, ASPP2 and iASPP protein expression was analyzed by immunofluorescence with corresponding antibodies in CRC (T) and adjacent non-cancerous controls (N). The average fluorescence intensity of the ASPP1, ASPP2 and iASPP proteins was examined. (C) ASPP1, ASPP2 and iASPP mRNA expression levels were investigated by reverse transcription-quantitative polymerase chain reaction. ${ }^{*} \mathrm{P}<0.05 ;{ }^{* *} \mathrm{P}<0.01 ;{ }^{* * *} \mathrm{P}<0.001$. ASPP, apoptosis-stimulating protein of p53; CRC, colorectal cancer; iASPP, inhibitor of ASPP; IOD, integrated optical density.

using RT-qPCR in both the TP53-positive and -negative groups. The results of the present study demonstrated that, compared with the TP53-negative group, only the levels of ASPP1 were declined in the TP53-positive group ( $\mathrm{P}<0.05$; Fig. 2D). However, no significant difference was observed in the expression levels of ASPP2 and iASPP between the two groups (Fig. 2E and F).

iASPP expression is correlated with the clinical course, and the size and extent of the primary tumor. In order to investigate the association between ASPP expression and disease pathological properties, the human CRC samples were classified into early (Stage I + II)/advanced (Stage III + IV) groups, as well as N0/Nx ( $\neq \neq 0)$ groups, M0/M1 groups, good/moderate/poor histological grade groups, and colon/rectal group based upon the Tumor-Node-Metastasis staging criteria of the American Joint Cancer Committee (22). T1, T2, T3 and T4 were based on the size and extent of the primary tumor (Table I). The ASPP
mRNA expression was determined by RT-qPCR in these groups (Fig. 1C). ASPP1 and ASPP2 expression did not exhibit any difference in the advanced stage group and the early stage group, however the iASPP level was markedly elevated in the advanced stage group compared with the early stage group (Fig. 3A-C). Furthermore, ASPP1 and ASPP2 expression also had no significant difference but the iASPP expression exhibited a gradient increase along with the enhancement of the tumor size and extent, where iASPP expression levels in the $\mathrm{T} 3$ and T4 group were markedly higher than those in the T1 or T2 group (Fig. 3D-F). However, no considerable changes in ASPP1, ASPP2, and iASPP expression were observed when $\mathrm{CRC}$ patients were classified based on regional lymph nodes and distant metastasis (Fig. 3G-L).

Significant correlation was observed between plasma CEA levels and ASPP $2 \mathrm{mRNA}$ expression in CRC samples. Since 
A
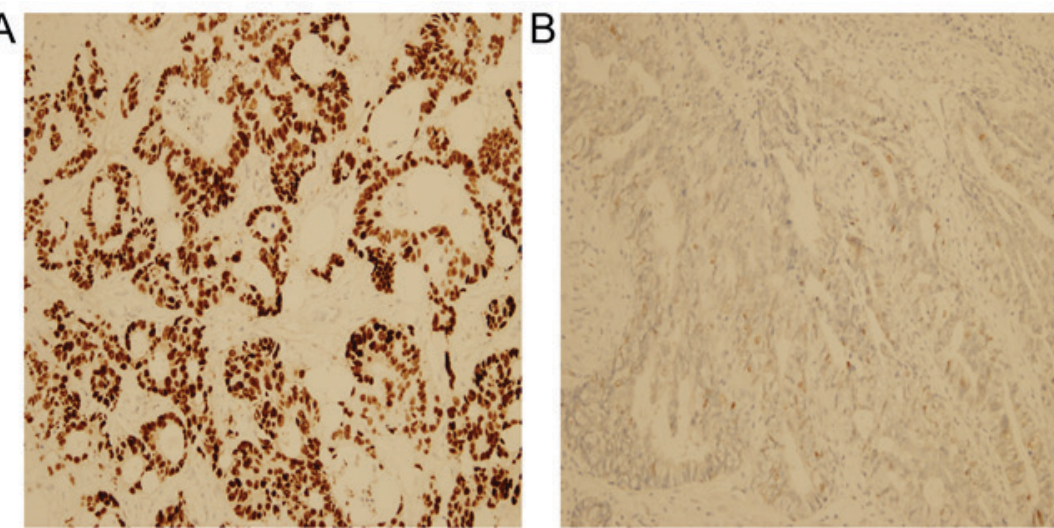

D

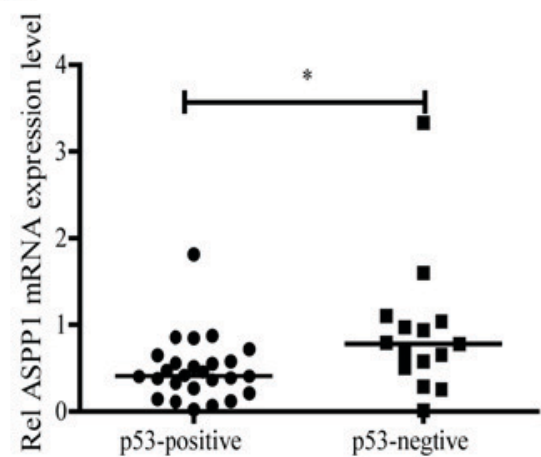

E

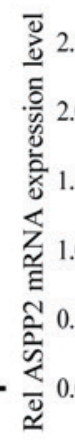

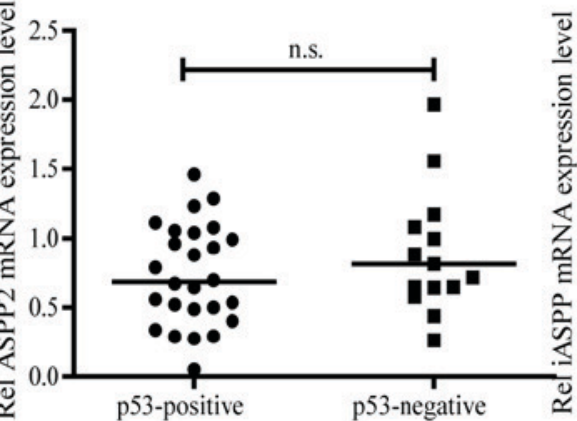

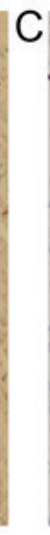

F
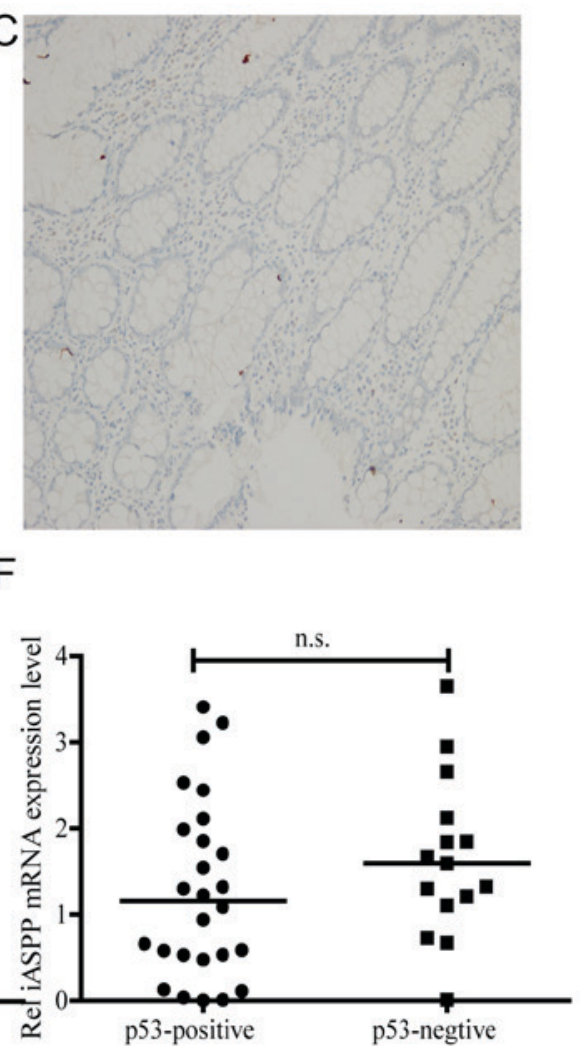

Figure 2. ASPP1 expression in TP53-positive and TP53-negative CRC samples. TP53 expression was verified by immunohistochemistry. Typical images of (A) TP53-positive and (B) TP53-negative human CRC group samples, and (C) an adjacent non-cancerous sample. The mRNA expression level of (D) ASPP1, (E) ASPP2 and (F) iASPP were analyzed by reverse transcription-quantitative polymerase chain reaction in TP53-positive and TP53-negative groups. *P<0.05, with comparisons shown by lines. ASPP, apoptosis-stimulating protein of p53; TP53, tumor protein p53; CRC, colorectal cancer; iASPP, inhibitor of ASPP; n.s., no significant difference.

the levels of CEA and AFP in the plasma remain valuable biomarkers for evaluating CRC progression (23-25), the potential association between ASPP mRNA expression and CEA or AFP concentrations in the samples was examined in the early and advanced groups (Fig. 4). No correlation was identified between the ASPP expression and AFP/CEA concentrations in the early or advanced groups (Fig. 4A-F). However, the ASPP2 level was negatively correlated with CEA expression in all the samples ( $\mathrm{r}=-0.0419 ; \mathrm{P}=0.0472$; Fig. 4G). Although certain changes in ASPP expression were detected in other observations, these differences were not statistically significant (data not shown). The mean value of CEA and AFP concentrations from all CRC tissues were 3.64 (range, 0.74-43.28) and 2.2 (range, 0-7.1) ng/ml, respectively.

\section{Discussion}

Previously, the alteration of ASPP expression during cancer development was reported in a number of tumor types $(17,26,27)$. However, few studies have focused on the role of ASPP in CRC development in either basic or clinical studies. Therefore, the present study aimed to investigate the role of ASPP in CRC progression and whether there is an association between ASPP and the pathology of the disease.

The preliminary data revealed that the protein and mRNA expression levels of ASPP1 and ASPP2 in human CRC specimens were significantly decreased compared with the corresponding adjacent non-cancerous controls. These observations are consistent with the results of other studies that reported low levels of ASPP in several tumor types, including breast, lung, non-small cell lung carcinoma, mesothelioma and leukemia (16,28-31). Therefore, these results indicated that the decreased expression of ASPP1 and ASPP2 weakened their anti-oncogene ability, causing the CRC cells to proliferate abnormally. The results of the present study demonstrated that the iASPP level in CRC samples was markedly augmented compared with that in the control samples (Fig. 1). This result is similar to those of other studies, in that iASPP is upregulated in numerous different tumors, including human breast carcinomas (32) and acute leukemia (AL) (33). iASPP, as a pro-tumorigenic factor, has been demonstrated to inhibit TP53 (34). Therefore, the abundant iASPP competed with the weakened ASPP1/ASPP2 to bind to TP53, resulting in the inhibition of the pro-apoptotic activity of TP53 and the promotion of CRC development (35). The detailed mechanism of the oncogenic cellular proliferation controlled by ASPPs is well established to be mediated through the TP53-mediated apoptotic regulation, but not through the TP53-involved cell cycle arrest (4,35).

TP53 has been reported to exist in wild-type and mutated forms in healthy mammals, which directly interferes with its tumor suppressing activity (36). Mutation of the amino acids, namely 178His, $181 \mathrm{Arg}$, 243Met, 247Arg, 248Arg and 273Arg, in the ASPP2 binding area of TP53 abolished its anti-oncogenic activity in a crystal structure analysis (36). In fact, these mutation sites are all frequently reported in human cancer (36). Notably, the mutated residues of 248Arg and 273Arg are involved in 
A

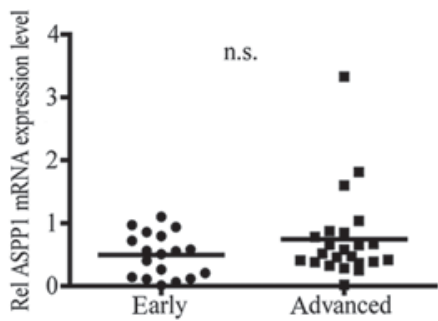

D

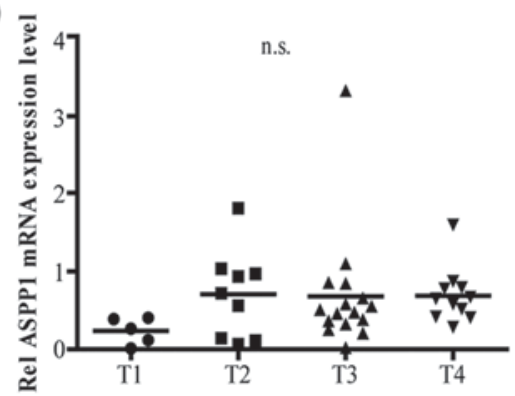

G

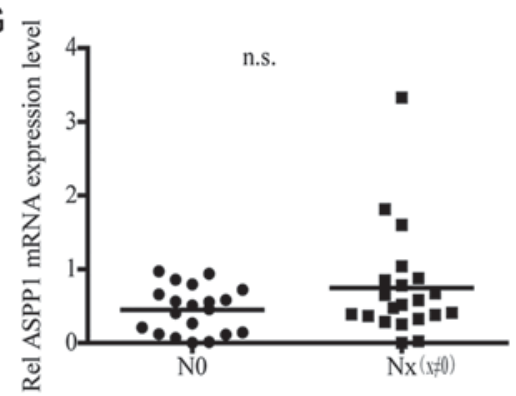

$\mathrm{J}_{\overline{\mathrm{g}}}$

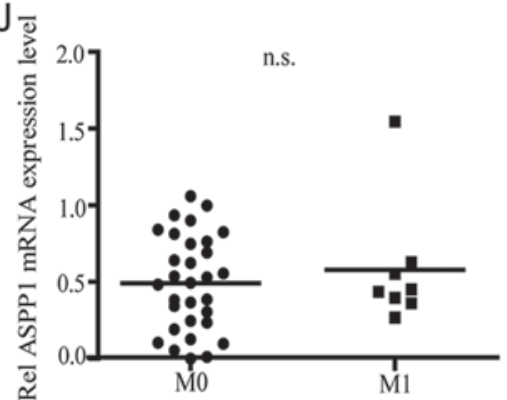

B

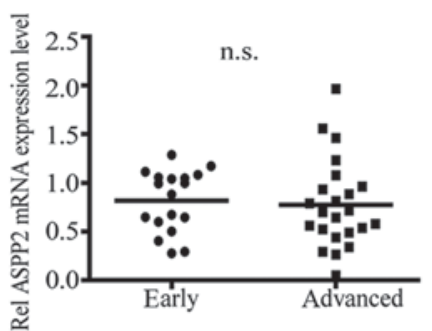

E

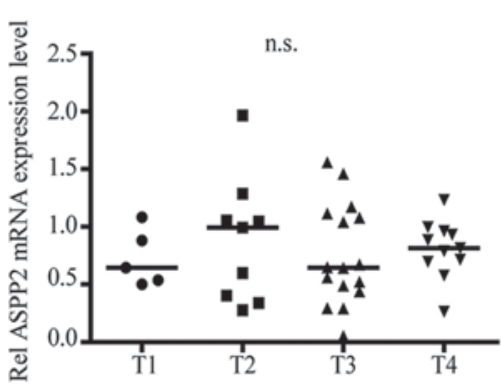

$\mathrm{H}$

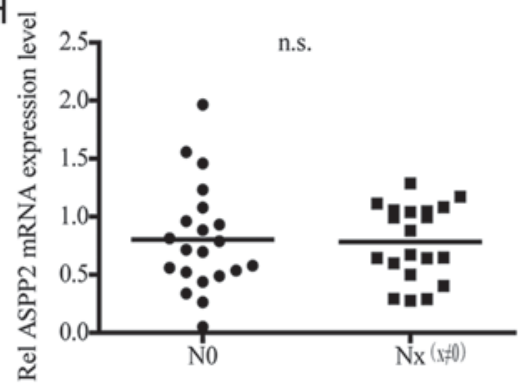

K

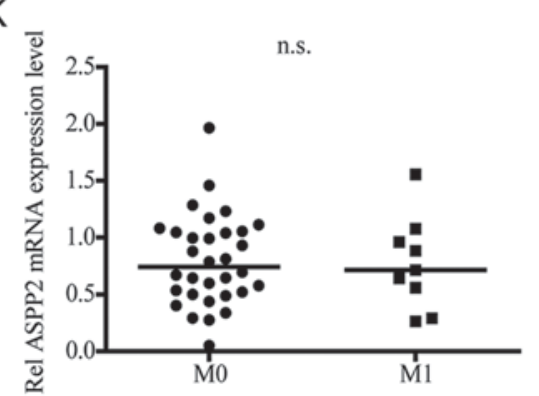

C

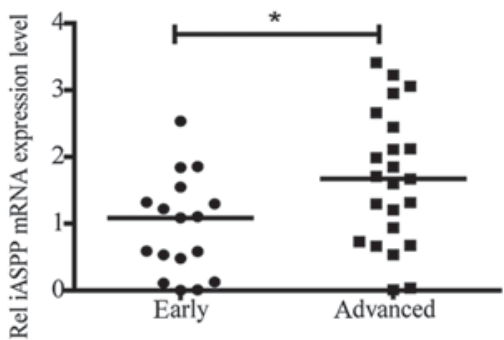

F

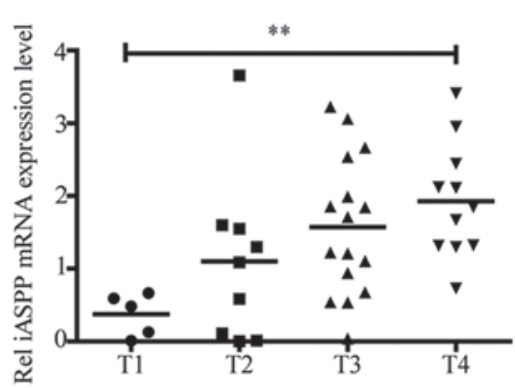

I

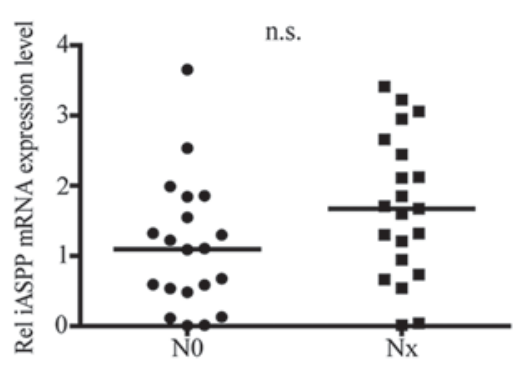

L

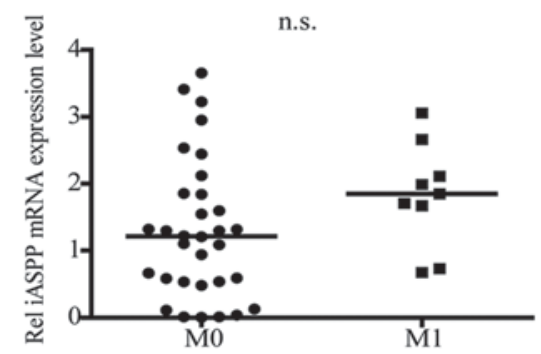

Figure 3. Associations between ASPP expression levels and the pathological grade of CRC malignancy, tumor topography, regional lymph node metastasis or distant metastases. The CRC samples were classified into multiple groups based on the different Tumor-Node-Metastasis staging system. The groups were classified as early or advanced groups based on the clinical course; T1, T2, T3 or T4 groups based on the on the size and/or extent of the primary tumor; N0 (negative) and Nx (positive) groups based on whether the cancer had migrated to the regional lymph nodes; and M0 (negative) or M1 (positive) based on whether there were distant metastases. The mRNA expression level of ASPP1, ASPP2 and iASPP were examined by reverse transcription-quantitative polymerase chain reaction. (A-C) The expression of ASPP1, ASPP2, iASPP in the early stage group and the advanced stage group. (D-F) The expression of ASPP1, ASPP2, iASPP in the T1 T4 groups. (G-I) The expression of ASPP1, ASPP2, iASPP in the N0 and Nx groups. (J-L) The expression of ASPP1, ASPP2, iASPP in the M0 and M1 groups. ${ }^{*} \mathrm{P}<0.05 ;{ }^{* *} \mathrm{P}<0.01$. ASPP, apoptosis-stimulating protein of p53; CRC, colorectal cancer; n.s., no significant difference.

DNA and ASPP2 binding. A total of 5-21\% of cancer patients harbor the mutated TP53 protein (8). Therefore, the presence and type of TP53 mutation is considered in patients with cancer, and whether it affects TP53 expression. It may be useful to determine whether or not ASPP expression is associated with the activity status of TP53 in human CRC. A study undertaken by Mori et al (17) was unable to identify any association between ASPP expression and TP53 status in lung cancer cell lines. Li et al (16) demonstrated that lower expression levels of ASPP1 and ASPP2 in non-small cell lung cancer (NSCLC) tumor tissues were more significantly associated with wild-type TP53 than with mutant TP53. In the present study, ASPP expression was analyzed in the TP53-positive group (a sample was considered TP53-positive if TP53 expression rate was $>30 \%$ compared with the healthy control using IHC analysis) and the TP53-negative group (TP53 expression rate $\leq 30 \%$ compared with the healthy control). The results demonstrated that the ASPP1 expression level was significantly lower in the TP53-positive group than in the TP53-negative group (Fig. 2D). This result was identical to the observations made by Li et al (16) in NSCLC tumor tissues. No notable changes were observed in the expression of either ASPP2 or iASPP (Fig. 2E and F). These data suggested that 

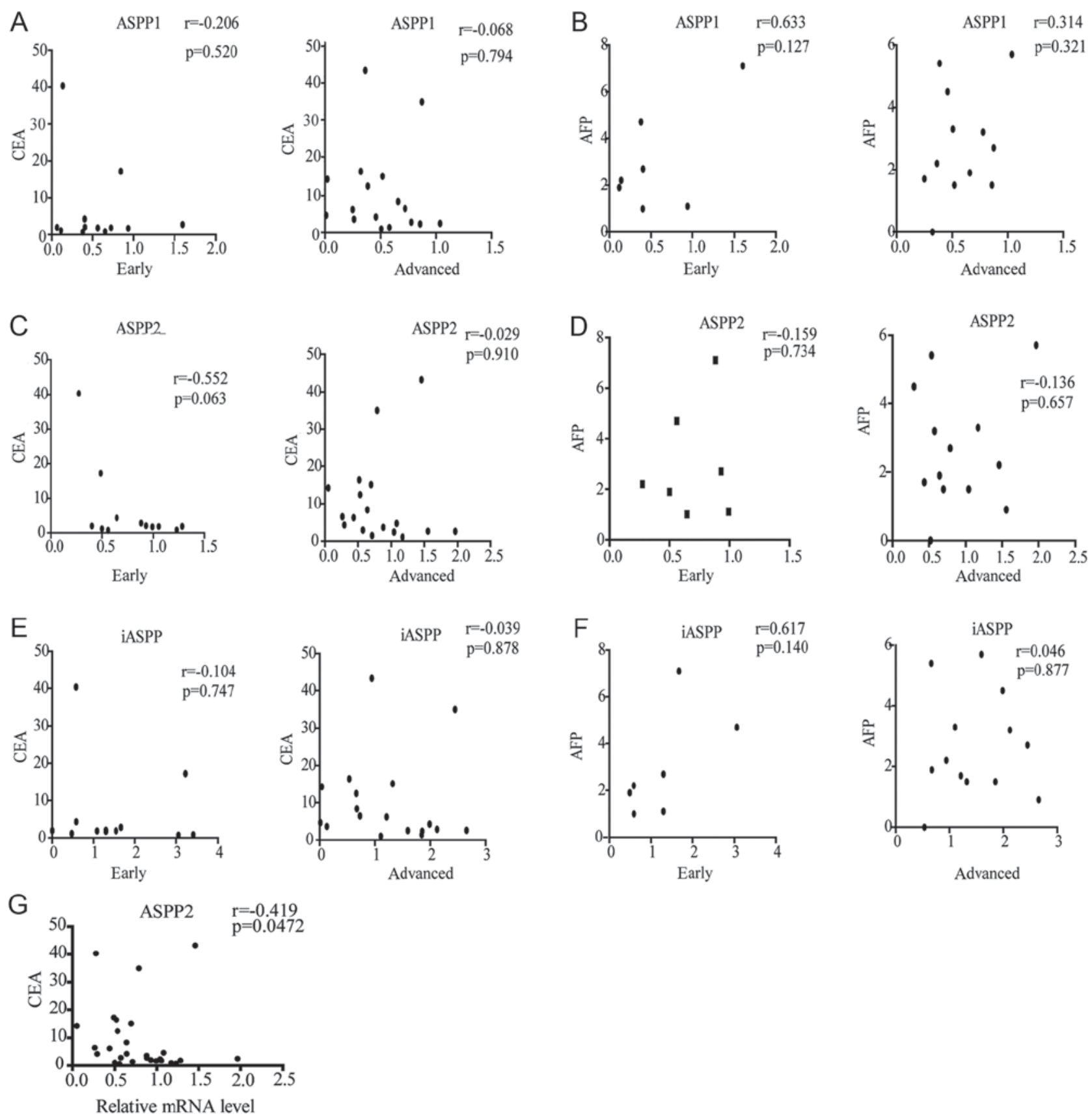

Figure 4. Pearson's correlation analysis of ASPP mRNA expression and the plasma concentration of CEA or AFP in early and advanced CRC groups. The mRNA expression of ASPP1, ASPP2 and iASPP were analyzed by reverse transcription-quantitative polymerase chain reaction. (A) Correlations between the level of ASPP1 and the plasma CEA concentration in the early and advanced CRC groups. (B) Correlations between the level of ASPP1 and the plasma AFP concentration in early and advanced CRC groups. (C) Correlations between the level of ASPP2 and the plasma CEA concentration in the early and advanced CRC groups. (D) Correlations between the level of ASPP2 and the AFP concentration in the early and advanced CRC groups. (E) Correlations between the level of iASPP and the plasma CEA concentration in the early and advanced CRC groups. (F) Correlations between the level of iASPP and the plasma AFP concentration in the early and advanced CRC groups. (G) Correlations between the level of ASPP2 and the plasma CEA concentration in all CRC samples were examined by linear regression analysis. ASPP, apoptosis-stimulating protein of p53; CEA, carcinoembryonic antigen; AFP, $\alpha$-fetoprotein; iASPP, inhibitor of ASPP; CRC, colorectal cancer.

the anti-oncogenic capability of the patients with CRC was adjusted through the homeostatic system following the aberrant proliferation of the CRC cells. Therefore, the higher expression of ASPP1 in patients with negative TP53 may be triggered in order to resist the pro-oncogenic forces. Since the antibody that was utilized in the IHC staining in the present study was unable to distinguish the wild-type from the mutated form of TP53, it is difficult to differentiate between the two and to analyze each one independently. However, the pro-oncogenic activity of TP53 is controlled by ASPP1 or ASPP2; therefore, the declined ASPP1 expression will primarily affect the pro-oncogenic ability of TP53 in this type of CRC.

A study undertaken by Zhang et al (34) revealed that iASPP expression was markedly higher in the blood cells of patients with acute leukemia (AL) than in those of healthy donors or patients with AL who had achieved complete remission. This observation suggested that iASPP may be employed as a marker of disease progression in AL (33). In addition, increased iASPP 
expression was also associated with a poor prognosis in FIGO IA2-IIA cervical adenocarcinoma (19). Low ASPP2 expression was reported to correlate with a poor prognosis in patients with diffuse large B-cell lymphoma (17) or breast cancer (26). Furthermore, metastatic breast cancer was revealed to be associated with a decreased ASPP2 expression compared with non-metastatic breast cancer (37). In order to observe whether or not the alteration of ASPP expression was associated with prognosis in patients with CRC, human CRC samples were divided into early and advanced groups based on the clinical course, $\mathrm{N} 0 / \mathrm{Nx}(\mathrm{x} \neq 0)$ groups, M0/M1 groups, good/moderate/poor histological grade groups, and colon/rectal groups based on TNM grading system, and the histological grade and tumor location. Notably, upregulated iASPP expression was revealed to be associated with the grade of malignancy in patients with CRC (Fig. 3C and F), but was not associated with metastasis to the regional lymph nodes (Fig. 3I), distant metastasis (Fig. 3L) or tumor location (data not shown). No significant association was observed between the expression of ASPP1 or that of ASPP2 in any of the groups.

In healthy patients, CEA is expressed at very low levels; however, certain types of cancer have been reported to have elevated levels of CEA in the serum. CEA, as a cell surface-anchored glycoprotein, is a functional ligand in colon carcinoma L-selectin and E-selectin, which may be involved in the metastatic dissemination of colon carcinoma cells (38-40). In the present study, no correlation was identified between plasma CEA concentration and APSS expression in early or advanced human CRC groups (Fig. 4). However, the alteration in the expression of ASPP2 in the entire CRC sample population exhibited a negative correlation with CEA concentration (Fig. 4G). This observation indicated that ASPP2 may be a valuable biomarker for CRC progression and a novel biomarker for serological cancer. It may be possible to make a preliminary diagnosis by detecting the level of ASPP2 expression in the serum. Additionally, in FIGO IA2-IIA cervical adenocarcinoma, the increased expression of iASPP was associated with a poor prognosis (26) and, in gestational trophoblastic disease, the downregulation of ASPP1 was correlated with clinical outcome (41). However, it is unclear if ASPP2 is correlated with clinical outcome and further investigation is required.

AFP is a major plasma protein during fetal development. Since it was revealed to be abnormally increased in certain types of tumor, AFP is frequently used as a diagnostic marker for several cancer types, including hepatocellular carcinomas (42), hepatoblastoma (43) and yolk sac tumors (44). The majority of tumors with increased AFP originate from the stomach, bile duct or pancreas (45), and upregulation of AFP is extremely rare in CRC due to the fact that the colorectum is initiated from the hindgut endoderm. As of yet, only 11 cases of CRC with increased-AFP have been reported in the early stages without distant metastases (45). The results of the present study demonstrated no correlation between AFP concentration and ASPP expression in early or advanced cases of CRC (Fig. 4). This may be attributed to the small sample size but, in order to validate these observations, a larger sample size is required.

In conclusion, the results of the present study confirmed that ASPP1 and ASPP2 expression were reduced, while iASPP expression was elevated in human CRC specimens, compared to the corresponding adjacent non-cancerous tissues. In addition, the upregulated iASPP may be used as a valuable biomarker for the grade of malignancy in human CRC. Finally, it was revealed that the upregulation of AFP is accompanied with an increase in iASPP expression in the early CRC group, compared with the advanced CRC group. These observations not only enrich the epidemic and clinical data of human CRC, but also provide valuable information for the development of novel drugs for the treatment of CRC, and for the improvement of CRC diagnosis, prognosis and therapy.

\section{Acknowledgements}

This study was supported by the National Natural Science Foundation of China (grant nos. 30972610 and 81273240), Jilin Province Science and Technology Agency (grant nos. 20160101037JC, 20170622009JC, 2017C021 and 2017J039), Norman Bethune Program of Jilin University (2012206), The fund of the State Key Laboratory of Kidney Diseases in PLA General Hospital.

\section{Funding}

No funding was received.

\section{Availability of data and materials}

The datasets generated and analyzed in the present study are included in this published article.

\section{Authors' contributions}

LW designed the experiment. LY, YL and XW performed the experiments and LY wrote the paper. YS, HH and CL processed the data. YJ participated in all of these processes, and revised the paper and approved the final version to be published.

\section{Ethics and consent to participate}

The study research proposal was approved by the Medical Ethics Committee of the First Hospital of Jilin University, and written informed consent was obtained from each patient.

\section{Consent for publication}

All participants provided written informed consent for the publication of the data.

\section{Competing interests}

The authors declare that they have no competing interests.

\section{References}

1. Haggar FA and Boushey RP: Colorectal cancer epidemiology: Incidence, mortality, survival, and risk factors. Clin Colon Rectal Surg 22: 191-197, 2009.

2. Shah TA: Colonic cancer; presentation and management in a surgical unit at allied hospital faisalabad. Prof Med J 23: 251-256, 2016.

3. Croce CM: Oncogenes and cancer. N Engl J Med 358: 502-511, 2008.

4. Trigiante G and Lu X: ASPP [corrected] and cancer. Nat Rev Cancer 6: 217-226, 2006. 
5. Chao C, Saito S, Kang J, Anderson CW, Appella E and Xu Y: p53 transcriptional activity is essential for p53-dependent apoptosis following DNA damage. EMBO J 19: 4967-4975, 2000.

6. Vousden KH and Lu X: Live or let die: The cell's response to p53. Nat Rev Cancer 2: 594-604, 2002.

7. Hollstein M, Sidransky D, Vogelstein B and Harris CC: p53 mutations in human cancers. Science 253: 49-53, 1991.

8. Starks AM, Martin DN, Dorsey TH, Boersma BJ, Wallace TA and Ambs S: Household income is associated with the p53 mutation frequency in human breast tumors. PLoS One 8: e57361, 2013.

9. Soussi T, Kato S, Levy PP and Ishioka C: Reassessment of the TP53 mutation database in human disease by data mining with a library of TP53 missense mutations. Hum Mutat 25: 6-17, 2005.

10. Vazquez A, Bond EE, Levine AJ and Bond GL: The genetics of the p53 pathway, apoptosis and cancer therapy. Nat Rev Drug Discov 7: 979-987, 2008.

11. Shi Y, Han Y, Xie F, Wang A, Feng X, Li N, Guo H and Chen D: ASPP2 enhances oxaliplatin (L-OHP)-induced colorectal cancer cell apoptosis in a p53-independent manner by inhibiting cell autophagy. J Cell Mol Med 19: 535-543, 2015.

12. Mak VC, Lee L, Siu MK, Wong OG, Lu X, Ngan HY, Wong ES and Cheung AN: Downregulation of ASPP2 in choriocarcinoma contributes to increased migratory potential through Src signaling pathway activation. Carcinogenesis 34: 2170-2177, 2013.

13. Schittenhelm MM, Illing B, Ahmut F, Rasp KH, Blumenstock G, Döhner K, Lopez CD and Kampa-Schittenhelm KM: Attenuated expression of apoptosis stimulating protein of p53-2 (ASPP2) in human acute leukemia is associated with therapy failure. PLoS One 8: e80193, 2013.

14. Song B, Bian Q, Zhang YJ, Shao CH, Li G, Liu AA, Jing W, Liu R, Zhou YQ, Jin G and Hu XG: Downregulation of ASPP2 in pancreatic cancer cells contributes to increased resistance to gemcitabine through autophagy activation. Mol Cancer 14: 177, 2015.

15. Meng WD, Chu RX, Wang BZ, Wang LP, Ma LL and Wang LX Infection à Helicobacter pylori et les expressions de l'apoptose liée à p53, PAES et PPEEE dans le cancer gastrique et des lésions précancéreuses. Pathol Biol 61: 199-202, 2013

16. Li S, Shi G, Yuan H, Zhou T, Zhang Q, Zhu H and Wang X: Abnormal expression pattern of the ASPP family of proteins in human non-small cell lung cancer and regulatory functions on apoptosis through p53 by iASPP. Oncol Rep 28: 133-140, 2012.

17. Mori $\mathrm{T}$, Okamoto $\mathrm{H}$, Takahashi $\mathrm{N}$, Ueda $\mathrm{R}$ and Okamoto $\mathrm{T}$ Aberrant overexpression of 53BP2 mRNA in lung cancer cell lines. FEBS Lett 465: 124-128, 2000.

18. Lossos IS, Natkunam Y, Levy R and Lopez CD: Apoptosis stimulating protein of $\mathrm{p} 53$ (ASPP2) expression differs in diffuse large B-cell and follicular center lymphoma: Correlation with clinical outcome. Leuk Lymphoma 43: 2309-2317, 2002.

19. Lu B, Guo H, Zhao J, Wang C, Wu G, Pang M, Tong X, Bu F, Liang A, Hou S, et al: Increased expression of iASPP, regulated by hepatitis B virus X protein-mediated NF- $\kappa$ B activation, in hepatocellular carcinoma. Gastroenterology 139: 2183-2194.e5, 2010.

20. Kim KH, Yang SS, Yoon YS, Lim SB, Yu CS and Kim JC: Validation of the seventh edition of the American joint committee on cancer tumor-node-metastasis (AJCC TNM) staging in patients with stage II and stage III colorectal carcinoma: Analysis of 2511 cases from a medical centre in Korea. Colorectal Dis 13 e220-e226, 2011

21. Livak KJ and Schmittgen TD: Analysis of relative gene expression data using real-time quantitative PCR and the 2(-Delta Delta C(T)) method. Methods 25: 402-408, 2001.

22. Sgroi DC, Teng S, Robinson G, LeVangie R, Hudson JR Jr and Elkahloun AG: In vivo gene expression profile analysis of human breast cancer progression. Cancer Res 59: 5656-5661, 1999.

23. Anzai H, Kazama S, Kiyomatsu T, Nishikawa T, Tanaka T, Tanaka J, Hata K, Kawai K, Yamaguchi H, Nozawa H, et al: Alpha-fetoprotein-producing early rectal carcinoma: A rare case report and review. World J Surg Oncol 13: 180, 2015.

24. Michl M, Stintzing S, Fischer von Weikersthal L, DeckerT, Kiani A, Vehling-Kaiser U, Al-Batran SE, Heintges T, Lerchenmueller C, Kahl C, et al: CEA response is associated with tumor response and survival in patients with KRAS exon 2 wild-type and extended RAS wild-type metastatic colorectal cancer receiving first-line FOLFIRI plus cetuximab or bevacizumab (FIRE-3 trial). Ann Oncol 27: 1565-1572, 2016

25. Huo YR, Glenn D, Liauw W, Power M, Zhao J and Morris DL: Evaluation of carcinoembryonic antigen (CEA) density as a prognostic factor for percutaneous ablation of pulmonary colorectal metastases. Eur Radiol 27: 128-137, 2017.
26. Kong F, Shi X, Li H, Li P, Yu J, Li X, Chen J, Sun Y and Jia Y: Increased expression of iASPP correlates with poor prognosis in FIGO IA2-IIA cervical adenocarcinoma following a curative resection. Am J Cancer Res 5: 1217, 2015.

27. Cobleigh MA, Tabesh B, Bitterman P, Baker J, Cronin M, Liu ML, Borchik R, Mosquera JM, Walker MG and Shak S: Tumor gene expression and prognosis in breast cancer patients with 10 or more positive lymph nodes. Clin Cancer Res 11: 8623-8631, 2005.

28. Vives V, Su J, Zhong S, Ratnayaka I, Slee E, Goldin R and Lu X: ASPP2 is a haploinsufficient tumor suppressor that cooperates with p53 to suppress tumor growth. Genes Dev 20: 1262-1267, 2006.

29. Samuels-Lev Y, O'Connor DJ, Bergamaschi D, Trigiante G, Hsieh JK, Zhong S, Campargue I, Naumovski L, Crook T and $\mathrm{Lu}$ X: ASPP proteins specifically stimulate the apoptotic function of p53. Mol Cell 8: 781-794, 2001

30. Liu ZJ, Lu X, Zhang Y, Zhong S, Gu SZ, Zhang XB, Yang X and Xin HM: Downregulated mRNA expression of ASPP and the hypermethylation of the 5'-untranslated region in cancer cell lines retaining wild-type p53. FEBS Lett 579: 1587-1590, 2005.

31. Mori S, Ito G, Usami N, Yoshioka H, Ueda Y, Kodama Y, Takahashi M, Fong KM, Shimokata K and Sekido Y: p53 apoptotic pathway molecules are frequently and simultaneously altered in nonsmall cell lung carcinoma. Cancer 100: 1673-1682, 2004.

32. Sullivan A and Lu X: ASPP: A new family of oncogenes and tumour suppressor genes. Br J Cancer 96: 196-200, 2007.

33. Bergamaschi D, Samuels Y, O'Neil NJ, Trigiante G, Crook T, Hsieh JK, O'Connor DJ, Zhong S, Campargue I, Tomlinson ML, et al: iASPP oncoprotein is a key inhibitor of $\mathrm{p} 53$ conserved from worm to human. Nat Genet 33: 162-167, 2003.

34. Zhang X, Wang M, Zhou C, Chen S and Wang J: The expression of iASPP in acute leukemias. Leuk Res 29: 179-183, 2005.

35. Liu ZJ, Zhang Y, Zhang XB and Yang X: Abnormal mRNA expression of ASPP members in leukemia cell lines. Leukemia 18: 880, 2004.

36. Lettre G, Kritikou EA, Jaeggi M, Calixto A, Fraser AG, Kamath RS, Ahringer J and Hengartner MO: Genome-wide RNAi identifies p53-dependent and -independent regulators of germ cell apoptosis in C. elegans. Cell Death Differ 11: 1198-1203, 2004.

37. Gorina $\mathrm{S}$ and Pavletich NP: Structure of the $\mathrm{p} 53$ tumor suppressor bound to the ankyrin and SH3 domains of 53BP2. Science 274: 1001-1005, 1996

38. Denoix PF: Enquete permanent dans les centres anticancereaux. Bull Inst Natl Hyg 1: 70, 1946

39. Thomas SN, Zhu F, Schnaar RL, Alves CS and Konstantopoulos K: Carcinoembryonic antigen and CD44 variant isoforms cooperate to mediate colon carcinoma cell adhesion to E- and L-selectin in shear flow. J Biol Chem 283: 15647-15655, 2008.

40. Konstantopoulos K and Thomas SN: Cancer cells in transit: The vascular interactions of tumor cells. Annu Rev Biomed Eng 11: 177-202, 2009.

41. Mak VC, Lee L, Siu MK, Wong OG, Lu X, Ngan HY, Wong ES and Cheung AN: Downregulation of ASPP1 in gestational trophpblastic disease: Correlation with hypermethylation, apoptopic activity and clinical outcome. Mod Pathol 24: 522-532, 2011.

42. Thomas SN, Tong Z, Stebe KJ and Konstantopoulos K: Identification, characterization and utilization of tumor cell selectin ligands in the design of colon cancer diagnostics. Biorheology 46: 207-225, 2009.

43. O'Conor GT, Tatarinov YS, Abelev GI and Uriel J: A collaborative study for the evaluation of a serologic test for primary liver cancer. Cancer 25: 1091-1098, 1970.

44. Smith CJ, Ajdukiewicz A and Kelleher PC: ConcanavalinA-affinity molecular heterogeneity of human hepatoma AFP and cord-serum AFP. Ann N Y Acad Sci 417: 69-74, 1983.

45. Norgaard-Pedersen R, Albrechtsen R and Teilum G: Serum alpha-foetoprotein as a marker for endodermal sinus tumour (yolk sac tumour) or a vitelline component of 'teratocarcinoma'. Acta Pathol Microbiol Scand A 83: 573-589, 1975.

This work is licensed under a Creative Commons Attribution-NonCommercial-NoDerivatives 4.0 International (CC BY-NC-ND 4.0) License. 